\title{
Route-choice support system for passengers in the face of unexpected disturbance of train operations
}

\author{
R. Tsuchiya ${ }^{1}$, Y. Sugiyama ${ }^{1}$, K. Yamauchi $^{1}$, K. Fujinami ${ }^{1}$, \\ R. Arisawa ${ }^{2} \&$ T. Nakagawa ${ }^{2}$ \\ ${ }^{1}$ Railway Technical Research Institute, Japan \\ ${ }^{2}$ East Japan Railway Company, Japan
}

\begin{abstract}
When train operation is disturbed due to an accident or natural disaster, passengers have to decide what they should do to get to their destination as early as possible. Unfortunately, however, this is not an easy task for passengers since sufficient information for decision-making is rarely provided to them. Therefore, we have developed a passenger support system which informs passengers of information on the optimal routes to their destinations by taking into consideration the predicted resumption time from the disturbance and the expected required time to each destination. More concretely, the system helps passengers decide whether to take the detour routes to their destinations or wait for the resumption of disturbed operation and continue their journey on the originally scheduled route. The system consists of an optimal route calculation engine and diversified man-machine interfaces, each of which is designed for a specific guidance application. Resumption time and required time to each destination are predicted based upon "resumption process model" whose parameters are determined by the statistical analysis of past data of train operation disturbances. The evaluation test of the prototype system is now being carried out by having a number of subjects in order to prove its effectiveness.

Keywords: route-choice support system, disturbance, passenger guidance.
\end{abstract}

\section{Introduction}

In order to increase customer satisfaction, it is very important for railway companies to provide appropriate guidance information to passengers when train 
operation is disturbed due to some accidents or natural disasters. When train operation is held up or disturbed, passengers have to decide what they should do to get to their destination as early as possible. Unfortunately, however, this is not an easy task for passengers since sufficient information for decision-making (i.e. time to resumption, estimated time to destination, etc) is rarely provided to them. Therefore, we have developed a route-choice support system which informs passengers of the optimal routes to their destinations by taking into consideration the predicted resumption time from the disturbance and the expected required time to each destination.

In the remainder of this paper, we will make an account of the background of this research, basic concepts of the system, followed by the design and implementation of the system. Also included is the result of the questionnaire survey which has been carried out by having a number of respondents randomly sampled from commuters in the urban area of Tokyo.

\section{Background of the research}

In Japan, there are several commercially available route-choice support systems for public transportation networks, which provide users with optimal travel plans to go to a specified destination. Few of them, however, can assist passengers with their decision-making when railway operations are disturbed. Some researchers of RTRI (Railway Technical Research Institute) have already proposed a passenger support system which calculates optimal detour routes to the destination when some of the railway lines are suspended. Although this feature is already supported by some of the route search engines commercially available, they can not help passengers decide whether they should take the detour route to the destination, or wait for the resumption of the suspended line and continue their journey on the originally scheduled route. In order to respond to these needs of passengers, more sophisticated decision support system is necessary.

Whether passengers can make appropriate decision depends on the correctness of the prediction as to when train operations will be resumed as well as the knowledge on the train operation rescheduling process. Therefore, we have developed a route-choice support system which informs passengers of information on the optimal routes to their destinations by taking into consideration the predicted resumption time from the disturbance and the estimated time to destination.

\section{Route-choice support system}

\subsection{Estimation of required time to destination}

In this research, we assumed that passengers in the face of unexpected disturbance of train operations want to know the fastest route to destination (figure 1). Based on this assumption, we propose a method which effectively realizes information provision that meets the demands of passengers. 


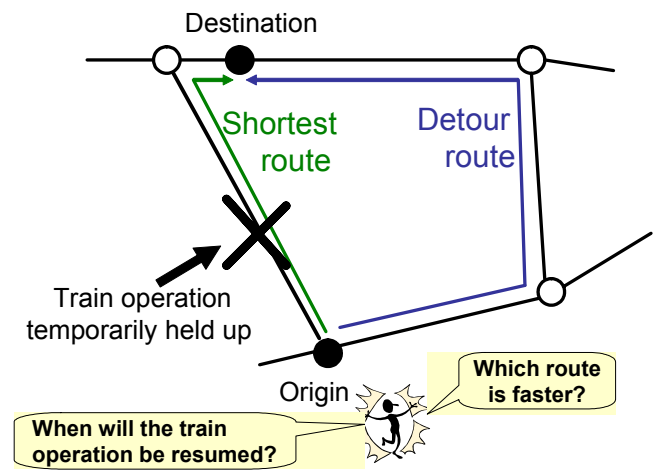

Figure 1: Passengers want to know which route is faster when train operations are disturbed.

We have devised a simplified resumption process model to estimate the required time between two adjacent stations. Required time is approximated by linear function as is depicted in figure 2 .

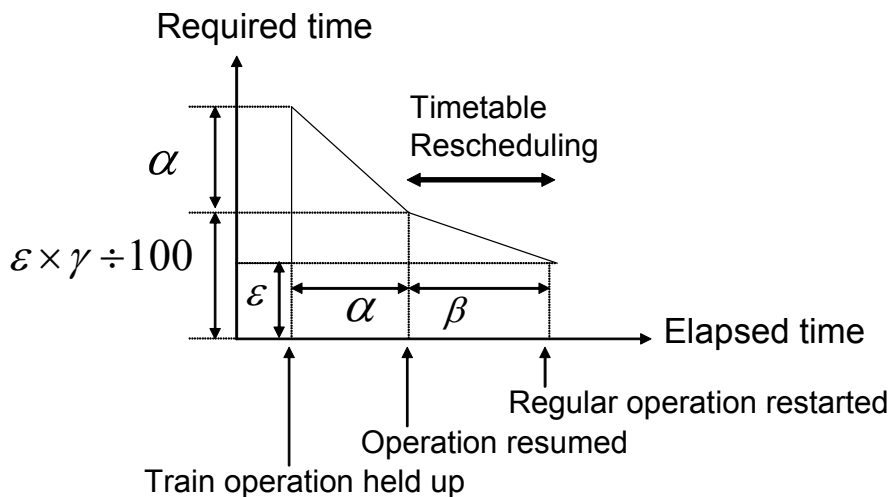

Figure 2: Simplified resumption process model for estimating required time between two adjacent stations.

$\alpha$ represents the elapsed time from occurrence of disturbance to the resumption of operation, whereas $\beta$ represents the elapsed time from the resumption of operation to the regular operation (i.e. operation with regular interval). Please note that even when the train operation is resumed, it has to undergo train rescheduling process, during which it often takes more time for passengers to go to their destinations than when trains are in regular operation. This is mainly due to the fact that in train operation rescheduling process, average speed and intervals of trains are often reduced. $\gamma$ represents the ratio 
which indicates how much extra time is needed in order to go from one station to another when train operation is in the rescheduling process. In our resumption process model, the value of each parameter is determined base on the statistical analysis of past data of train operation disturbances. These values are default values which can be modified by human operators.

\subsection{Presenting guidance information to many and unspecified passengers}

Guidance information can be provided to passengers in two different ways. One method is to present guidance information to many and unspecified passengers, typically at stations by using large screen displays. Another method is to deliver personalized guidance messages to passengers' portable terminals including cellular phones. One of the difficulties in presenting guidance information to many and unspecified passengers is that unlike personalized information provision, it is impossible to customize the presentation based on a passenger's destination, his/her knowledge level on the detour route, his/her ability to use electronic devices, etc. Usability of the system heavily depends on the way how the guidance information is presented to passengers and how effective it is when they make travel decisions. In this research, therefore, we have devised two different user interfaces for presenting guidance information for many and unspecified passengers, which will be evaluated to find the best way. One user interface which we have developed is based on the map of railway network for urban area of Tokyo, which we often see at stations or on board trains. Appropriateness for taking a detour route, which depends on the destination, is classified by colour per station on the map (figure 3 ).

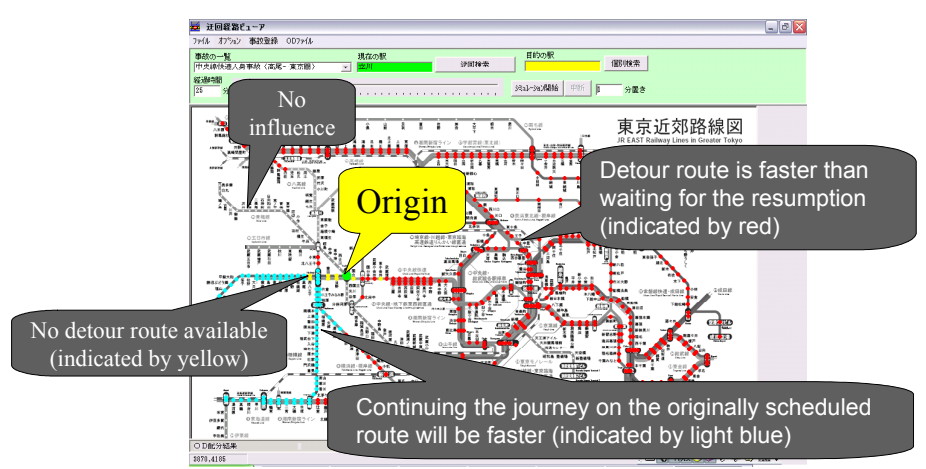

Figure 3: Appropriateness for taking a detour route.

As is shown in figure 3, every station is attached one of the four colours (i.e. red, yellow, light blue and colourless). The configuration of colours changes every minute so that up-to-date information is presented to passengers.

Table 1 briefly describes the meaning of colours attached to each station. 
Table 1: Colors and their meanings.

\begin{tabular}{|c|c|l|}
\hline categories & colors & \multicolumn{1}{c|}{ meanings } \\
\hline $\begin{array}{c}\text { Detour route } \\
\text { recommended }\end{array}$ & red & $\begin{array}{l}\text { Detour route is advantageous over } \\
\text { originally scheduled route }\end{array}$ \\
\hline No detour route & yellow & $\begin{array}{l}\text { No detour route available. You have } \\
\text { no choice but wait for the resumption }\end{array}$ \\
\hline $\begin{array}{c}\text { Originally } \\
\text { scheduled route } \\
\text { will be better }\end{array}$ & light blue & $\begin{array}{l}\text { Detour route available but waiting for } \\
\text { resumption and continue the journey } \\
\text { on the originally scheduled route will } \\
\text { be better }\end{array}$ \\
\hline No influence & colorless & No influence of disturbance \\
\hline
\end{tabular}

"Detour route recommended" (red) indicates that the destination (station) can be reached earlier by taking detour route than by originally scheduled route. "No detour route" (yellow) indicates that there are no detour routes to the destination and passengers have to wait for the resumption of the operation and continue their journey on the originally scheduled route. "Wait for the resumption" (light blue) indicates that passengers will be able to arrive at the destination earlier by waiting for the resumption and take the originally scheduled route than by taking the detour route.

This type of presentation can inform passengers whether taking the detour route is advantageous over taking the originally scheduled route by waiting for resumption. It does not, however, tell passengers how they can go to the destination by using the detour route. Therefore we have devised a way of presenting more detailed information on the detour route for restricted number of destinations so that passengers going to those major destinations will be provided with more detailed guidance (figure 4).

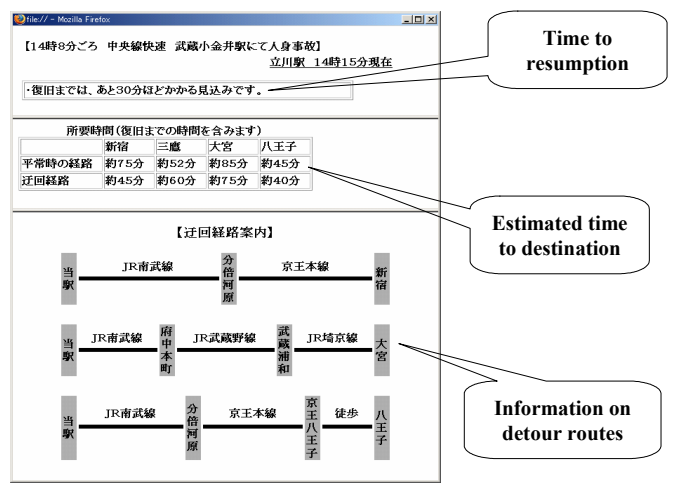

Figure 4: Screen which indicates estimated time to destination and detour route details. 


\subsection{Application to personalized guidance}

Although our main target is information provision for many and unspecified passengers, our method can also be applied to personalized guidance. Figure 5 is an example of messages delivered to a portable terminal which includes time to resumption, estimated time to destination as well as detour route details [1].

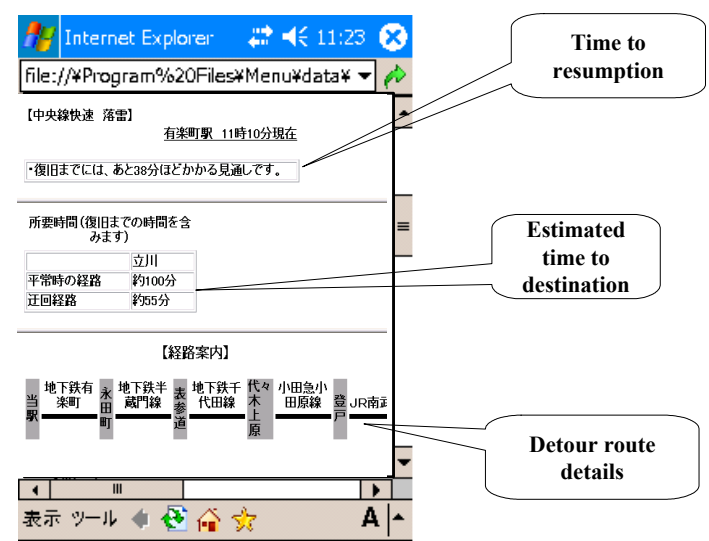

Figure 5: $\quad$ Example of guidance message delivered to portable terminal.

\subsection{System implementation}

The system that we have developed consists of optimal route calculation engine and several applications which, by cooperating with the engine, present guidance information in a variety of ways. Typical application of the system might include, as is described in previous sections, variable signs for many and unspecified passengers at stations, support systems for station staffs engaged in passenger guidance as well as personalized guidance systems using passengers' portable terminals. Therefore we have developed a general purpose optimal route calculation engine which include detour route calculation feature (figure 6).

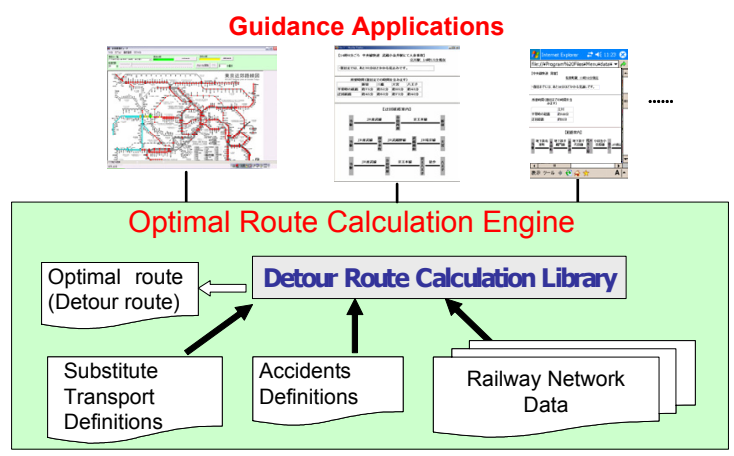

Figure 6: Optimal route calculation engine. 
Core functionalities of the system are realized by the detour route calculation library which calculates optimal routes from a specified station to all JR (Japan Rail) stations, taking into consideration disturbances of railway operation. Input data to the system consists of railway network data, substitutable transport definitions and accidents definitions. Railway network data covers nation-wide JR lines as well as lines belonging to all private railways. In Japan, most of the railway companies are in agreement with each other on the mutual provision of substitute transport in case some of the railway companies encounter disturbance of train operations and therefore cannot provide sufficient transport capacity. Since these requests are usually accepted, passengers can take detour routes without any extra charge. Input data "substitution transport definitions" represents basic patterns which specify which railway lines should be used as substitution transport depending on the place where a train operation disturbance takes place.

Input data "accidents definitions" defines parameters (i.e. $\alpha, \beta, \gamma$ ) related to accidents which take place in some of the JR lines (figure 7). Although the default values of parameters are determined by statistical analysis of past data of train operations disturbances, users (i.e. commanders or station staffs of each railway) can modify the values of parameters when necessary.

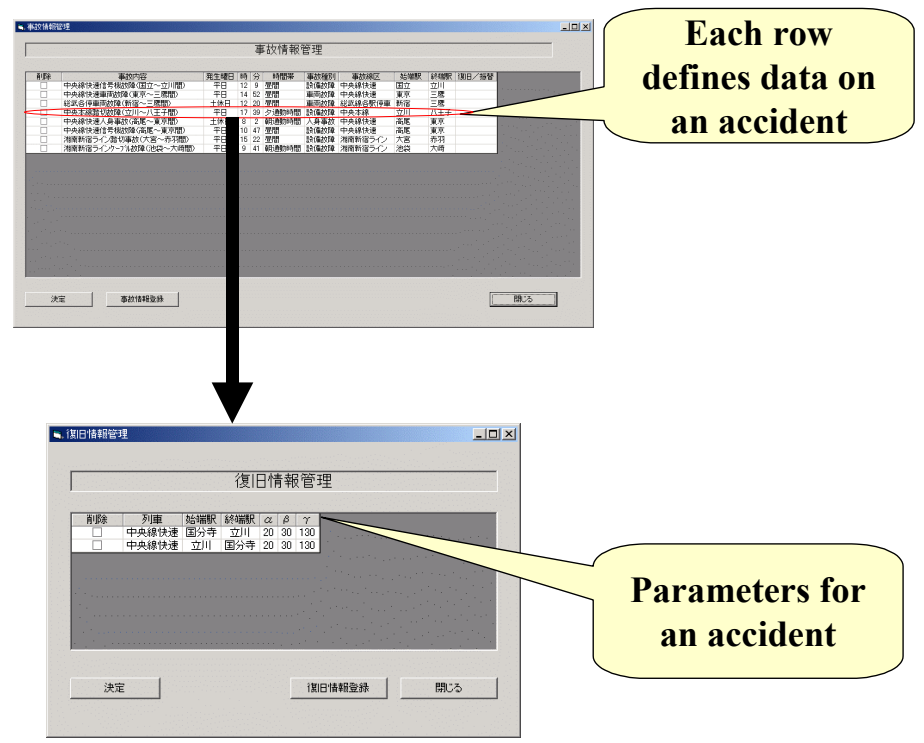

Figure 7: Accidents data definitions.

In order to find the optimal (i.e. shortest) route to a destination, the system has to calculate expected time to destination for several candidate routes. In our system, the expected time to a destination via a specified route is calculated by accumulating the expected time between two adjacent stations which belong to the route. 
If the candidate route does not contain disturbed railway lines, the weight of the link which correspond to a pair of adjacent stations are specified based on the average running time between these two stations. Whereas, when the candidate route contains disturbed railway lines, the system estimate the running time between the stations based on the resumption process model depicted in figure 2 and set the corresponding value as the weight of the link. When calculating the estimated running time between two adjacent stations, the system takes into consideration the elapsed time to get to either of the two stations which is also estimated by the model. For example, if you are going from a station which is far away enough from the disturbed railway line, there will be little effect on your itinerary even if it contains the disturbed line.

\section{Evaluation}

The optimal-route calculation engine which we have developed can calculate optimal routes from a specified station to all JR stations in Japan in a few seconds by using a general purpose office PC. The performance that we have achieved with our calculation engine is sufficiently high to realize the information update interval which we expect to be one minute.

We have so far demonstrated our system at several expositions and rail-related conferences and received favorable reactions from participants. They highly evaluated its effectiveness as a tool to help passengers' decision-making when they encounter train operation disturbances. With the increasing needs of information provision for passengers when train operations are disturbed, several railway companies are currently trying to provide passengers with more information in a timely manner even if it contains uncertainty. For example, some of the railway companies in Tokyo urban areas started to provide information related to the time when the train operation is expected to be resumed. Although this kind of information contains some uncertainty and is not always true, they seem to be accepted favorably by most of the passengers.

Providing information timely even though it contains uncertainty may be justified by the questionnaire survey which we have carried out as a part of this research. We asked the respondents about their attitudes toward the provision of uncertain information as to when the train operations are expected to be resumed. More concretely, we asked how similar their attitudes are to the following two contrastive attitudes.

A: I expect that information be provided timely, even though it contains some uncertainty

B: I expect that information be provided only when it is certain. Uncertain information may cause confusion.

According to the result of the survey, $94 \%$ of the respondents' attitudes are similar to that of A.

We are currently carrying out the test of the prototype system by having a number of subjects randomly sampled from commuters in Tokyo urban area in order to evaluate the effectiveness of the system, appropriateness of presentation as well as its acceptability as a tool to help passengers' decision-making. 


\section{Conclusions}

We have developed a route-choice support system which helps passengers make appropriate decision when train operations are disturbed. The system helps passengers decide whether to take the detour routes to their destinations or wait for the resumption of disturbed operation and continue their journey on the originally scheduled route. The system consists of an optimal route calculation engine and diversified man-machine interfaces, each of which is designed for a specific guidance application. Typical application of the system might include variable signs for many and unspecified passengers at stations, support systems for station staffs engaged in passenger guidance, personalized guidance systems using passengers' portable terminals, etc.

We have so far demonstrated our system at several expositions and railrelated conferences and received favorable reactions. They highly evaluated its effectiveness as a tool to help passengers' decision-making when they encounter train operation disturbances.

The evaluation test of the prototype system is now being carried out by having a number of subjects and its result will be reported soon.

\section{References}

[1] Tsuchiya, R., Nozue, M.: "Supporting Intermodal Travelers by Agent-based Information Integration," $8^{\text {th }}$ World Congress on Railway Research (WCRR 2006), 2006 (to appear)

[2] Tsuchiya,R., Sugiyama,Y., Yamauchi,K., Fujinami,K., Arisawa,R., Nakagawa,T.: "Route-choice Support System for Passengers Based on Predicted Resumption Time from Unexpected Disturbance of Train Operations,”, RTRI Report, Vol. 20, No.2, Feb. 2006 (in Japanese) 